\title{
Quantitative Interrelation between Atractylenolide I, II, and III in Atractylodes japonica Koidzumi Rhizomes, and Evaluation of Their Oxidative Transformation Using a Biomimetic Kinetic Model
}

\author{
Jung-Hoon Kim, ${ }^{*}+\odot$ Yuvin Lee, ${ }^{\ddagger}$ Guemsan Lee, ${ }^{\S}$ Eui-Jeong Doh, ${ }^{\S, \|}$ and Seungwoo Hong, ${ }^{*} \$ \odot$ \\ ${ }^{\dagger}$ Division of Pharmacology, School of Korean Medicine, Pusan National University, 50612 Yangsan, Republic of Korea \\ ${ }^{\ddagger}$ Department of Chemistry, The Research Institute of Natural Sciences, Sookmyung Women’s University, 04310 Seoul, Republic of \\ Korea \\ ${ }^{\S}$ Department of Herbology, College of Korean Medicine, and "Research Center of Traditional Korean Medicine, Wonkwang \\ University, 54538 Iksan, Republic of Korea
}

Supporting Information

\begin{abstract}
Analytical methods based on ultraperformance liquid chromatography/ion-trap mass spectrometry (UPLC/ion-trap MS) were developed for quantification of atractylenolide I, II, and III in the methanol extract of Atractylodes japonica rhizomes with a $\mathrm{C}_{18}$ column in an acidified water/acetonitrile gradient eluent in an LC system, and ion-trap MS coupled with electrospray ionization was employed under positive-ion mode. The three atractylenolides were quantified in all A. japonica samples, and the content of atractylenolide I, II, and III showed a significant correlation to each other. Such high correlation was explained by the mechanistic insights into the biosynthetic pathway of atractylenoide III and I from atractylenoide II by using the biomimetic cytochrome $\mathrm{P} 450$ model, $[\mathrm{Fe}(\mathrm{tmp})]\left(\mathrm{CF}_{3} \mathrm{SO}_{3}\right)$ $(\mathrm{tmp}=$ meso-tetramesitylporphyrin). Atractylenolides could be transformed by oxidation via the oxidative enzyme in the A. japonica plant. The present study first reports the first oxidative transformation of atractylenolides using the heme iron model complex.
\end{abstract}

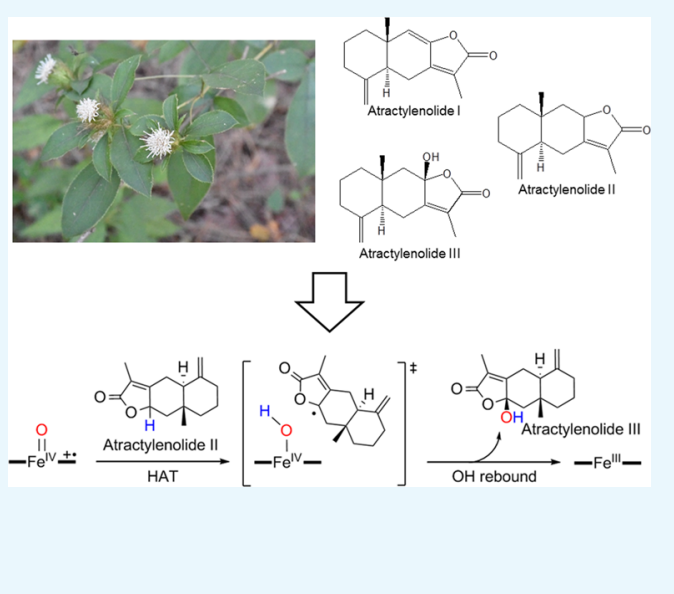

\section{INTRODUCTION}

The plant Atractylodes japonica Koidzumi is distributed in Korea, northeastern China, and Japan, and its dried rhizome has been used to improve gastrointestinal mobility by the induction of distal colon contraction and increasing colonic transit time. ${ }^{1,2}$ Atractylenolide I, II, and III, which are sesquiterpene-type bioactive compounds in $A$. japonica rhizomes, also exhibit gastrointestinal activities both pharmacologically and clinically: atractylenolide I has been shown to offer improved treatment of gastric cancer and gastric cancer cachexia, and the stimulation of intestinal epithelial cell migration and proliferation, and atractylenolide III has shown gastroprotective activity against acute gastric ulcers. ${ }^{3-6}$ Hence, it is thought that atractylenolides in A. japonica rhizomes play a key role in the treatment of gastrointestinal diseases.

Many analytical methods have been developed for the simultaneous determination or quantification of atractylenolides in Atractylodes rhizomes. High-performance liquid chromatography (HPLC)-diode array detection methods have been widely employed for the quantification of atractylenolides II and III in Atractylodes macrocephala rhizomes and atractylenolides I and III in A. japonica rhizomes. $^{7-9}$ Gas chromatography-mass spectrometry (MS) has also been used for the determination of atractylenolides I and III in A. macrocephala rhizomes. ${ }^{10,11}$ Being regarded as sensitive and efficient techniques, the $\mathrm{LC} /$ triple quadrupole MS method was adopted to identify and quantify atractylenolides in the rhizomes of $A$. japonica, A. macrocephala, Atractylodes lancea, and Atractylodes chinensis. ${ }^{12}$ Recently, the identification and quantification of atractylenolide I and III in the A. macrocephala rhizome by using LC/ion-trap MS has been reported. ${ }^{13}$ Ion-trap MS can be used to isolate and accumulate ions through the application of characteristic electrodes and oscillating electric fields, and the technique is advantageous for inducing ion fragmentation and accumulation with higher resolution by collision-induced decomposition. ${ }^{14-16}$ By using these advantages, ion-trap MS has been applied for the identification and quantification of chemical components in herbal medicines. ${ }^{17-19}$ Nevertheless, the quantitative analysis of atractylenolides I, II, and III in $A$. japonica rhizomes using LC/MS, especially ion-trap MS, has not been reported.

Received: August 13, 2018

Accepted: October 23, 2018

Published: November 5, 2018 
Table 1. Intra- and Interday Precisions of Three Marker Compounds in the Methanol Extract of A. japonica Rhizomes

\begin{tabular}{|c|c|c|c|c|c|c|c|}
\hline \multirow[b]{2}{*}{ compound } & \multirow[b]{2}{*}{$\begin{array}{l}\text { initial concentration } \\
\qquad(\mu \mathrm{g} / \mathrm{mL})\end{array}$} & \multicolumn{3}{|c|}{ intraday $(n=3)$} & \multicolumn{3}{|c|}{ interday $(n=3)$} \\
\hline & & $\begin{array}{l}\text { detected concentration } \\
(\mu \mathrm{g} / \mathrm{mL})\end{array}$ & $\begin{array}{l}\mathrm{RSD}^{a} \\
(\%)\end{array}$ & $\begin{array}{l}\text { accuracy } \\
(\%)\end{array}$ & $\begin{array}{l}\text { detected concentration } \\
(\mu \mathrm{g} / \mathrm{mL})\end{array}$ & $\begin{array}{c}\mathrm{RSD} \\
(\%)\end{array}$ & $\begin{array}{l}\text { accuracy } \\
(\%)\end{array}$ \\
\hline \multirow{3}{*}{ atractylenolide III } & 0.94 & 0.86 & 12.32 & 91.74 & 0.86 & 12.03 & 91.42 \\
\hline & 3.75 & 4.27 & 2.10 & 113.94 & 4.34 & 4.64 & 115.70 \\
\hline & 15.00 & 15.87 & 4.05 & 105.82 & 16.26 & 5.73 & 108.42 \\
\hline \multirow[t]{3}{*}{ atractylenolide II } & 0.63 & 0.59 & 1.97 & 93.66 & 0.52 & 10.62 & 82.85 \\
\hline & 2.50 & 2.72 & 2.52 & 108.97 & 2.73 & 4.77 & 109.10 \\
\hline & 10.00 & 10.47 & 4.89 & 104.66 & 11.05 & 7.82 & 110.52 \\
\hline \multirow[t]{3}{*}{ atractylenolide I } & 0.94 & 0.86 & 5.89 & 91.29 & 0.81 & 9.34 & 86.93 \\
\hline & 3.75 & 4.07 & 2.50 & 108.46 & 4.05 & 3.19 & 107.89 \\
\hline & 15.00 & 15.56 & 5.03 & 103.73 & 16.04 & 6.56 & 106.95 \\
\hline
\end{tabular}

Table 2. Recovery of Three Marker Compounds in the Methanol Extract of $A$. japonica Rhizomes $(n=3)$

\begin{tabular}{|c|c|c|c|c|c|}
\hline compound & initial concentration $(\mu \mathrm{g} / \mathrm{mL})$ & spiked concentration $(\mu \mathrm{g} / \mathrm{mL})$ & detected concentration $(\mu \mathrm{g} / \mathrm{mL})$ & recovery $(\%)$ & $\operatorname{RSD}^{a}(\%)$ \\
\hline \multirow[t]{3}{*}{ atractylenolide III } & 8.22 & 1.88 & 9.90 & 89.56 & 9.12 \\
\hline & & 3.75 & 12.00 & 100.64 & 13.95 \\
\hline & & 7.50 & 15.87 & 101.93 & 8.91 \\
\hline \multirow[t]{3}{*}{ atractylenolide II } & 8.20 & 1.25 & 9.36 & 93.15 & 12.29 \\
\hline & & 2.50 & 10.61 & 96.45 & 11.19 \\
\hline & & 5.00 & 13.11 & 98.34 & 6.66 \\
\hline \multirow[t]{3}{*}{ atractylenolide I } & 11.20 & 1.88 & 12.76 & 83.50 & 12.74 \\
\hline & & 3.75 & 14.49 & 87.75 & 13.47 \\
\hline & & 7.50 & 18.36 & 95.51 & 7.25 \\
\hline
\end{tabular}

Meanwhile, in cytochromes P450 (CYP450) is present in plants, high-valent iron(IV)-oxo porphyrin $\pi$-cation radicals, referred to as compound I ( $\mathrm{Cpd} \mathrm{I}$ ), have been proposed as reactive intermediates mainly involved in the biosynthetic pathway of sesquiterpenes. ${ }^{20}$ For example, the hydroxylation of alkanes by Cpd I is initiated by a hydrogen atom abstraction (HAT) step, followed by an oxygen rebound step. ${ }^{21}$ However, kinetic and mechanistic insights into the hydroxylation of atractylenolides by Cpd I have not yet been evidenced.

In the present study, three marker compounds of the $A$. japonica rhizome, atractylenolides I, II, and III, were quantified in the methanol extract of $A$. japonica samples obtained from different locations in Korea and China using LC/ion-trap MS. Moreover, the correlation between the amounts of the three marker compounds in A. japonica samples was evaluated by calculating Pearson's correlation coefficient. Moreover, we performed kinetic and mechanistic studies on the hydroxylation of atractylenolides II by the iron(IV)-oxo porphyrin $\pi$ cation radical complex, $\left[\left(\mathrm{tmp}^{+\bullet}\right) \mathrm{Fe}^{\mathrm{IV}}(\mathrm{O})\right]^{+}$.

\section{RESULTS AND DISCUSSION}

2.1. Optimization of Chromatographic Conditions. Optimal conditions for chromatographic analysis were established by comparing the column, mobile phase, mobile phase modifier, and flow rate to separate and detect the three marker compounds and the internal standard (IS) in $A$. japonica samples. A Thermo Hypersil GOLD $\mathrm{C}_{18}$ column (2.1 $\mathrm{mm} \times 100 \mathrm{~mm}, 1.9 \mu \mathrm{m})$ was chosen because it afforded improvement of the peak shape and better resolution within 15 min, compared with a Thermo Hypersil GOLD $\mathrm{C}_{18}$ column $(2.1 \mathrm{~mm} \times 50 \mathrm{~mm}, 1.9 \mu \mathrm{m})$. The mobile phase consisted of water and acetonitrile, with $0.1 \%$ formic acid being added for better ionization of the marker compounds and thus high signal intensity, as previously reported. ${ }^{22}$ The flow rate of the gradient elution was $250 \mu \mathrm{L} / \mathrm{min}$ as an optimal eluent for ionization of the marker compounds and the IS in electrospray ionization (ESI) mode. Tandem mass scans were conducted simultaneously, and the peak of each marker compound was baseline separated (Figure S1, Supporting Information).

2.2. Optimization of Mass Spectrometric Conditions. To determine the optimal ESI conditions for the ion-trap MS measurements, each marker compound was directly injected into MS in both negative and positive ionization modes. Higher signal intensity and good sensitivity were observed in positive ionization mode than in negative ionization mode, as shown previously. ${ }^{13,22-24}$ Thus, positive-ion mode was selected for analysis of the three marker compounds in the A. japonica samples. Different conditions such as sheath gas $(30,40$, and 50 arb units), auxiliary gas (10,20, and 30 arb units), capillary temperature $\left(270-300{ }^{\circ} \mathrm{C}\right)$, and capillary voltage $(20,30$, and $40 \mathrm{~V}$ ) were compared. The highest intensity of protonated molecular ions and fragmented ions was obtained under the following conditions: sheath gas, 50 arb units; auxiliary gas, 20 arb units; capillary temperature, $280{ }^{\circ} \mathrm{C}$; and capillary voltage, $30.0 \mathrm{~V}$.

The protonated molecular ion $[\mathrm{M}+\mathrm{H}]^{+}$of atractylenolide III $(m / z 249.00)$ lost an $\mathrm{H}_{2} \mathrm{O}$ molecule and was converted into a fragment ion $(m / z 231.03)$, which was observed with the highest intensity. The protonated molecular ion of atractylenolide II $(m / z 233.00)$ also lost an $\mathrm{H}_{2} \mathrm{O}$ molecule and was converted into a fragment ion with $\mathrm{m} / z$ 215.07, which was observed with the highest intensity. Unlike the two compounds discussed above, the protonated molecular ion of atractylenolide I $(\mathrm{m} / z 231.00)$ was mainly converted into a fragment ion $(m / z$ 185.09). The protonated molecular ion of bergapten (IS, $m / z$ 217.00) lost a $\mathrm{CH}_{3}$ molecule and was converted into a 

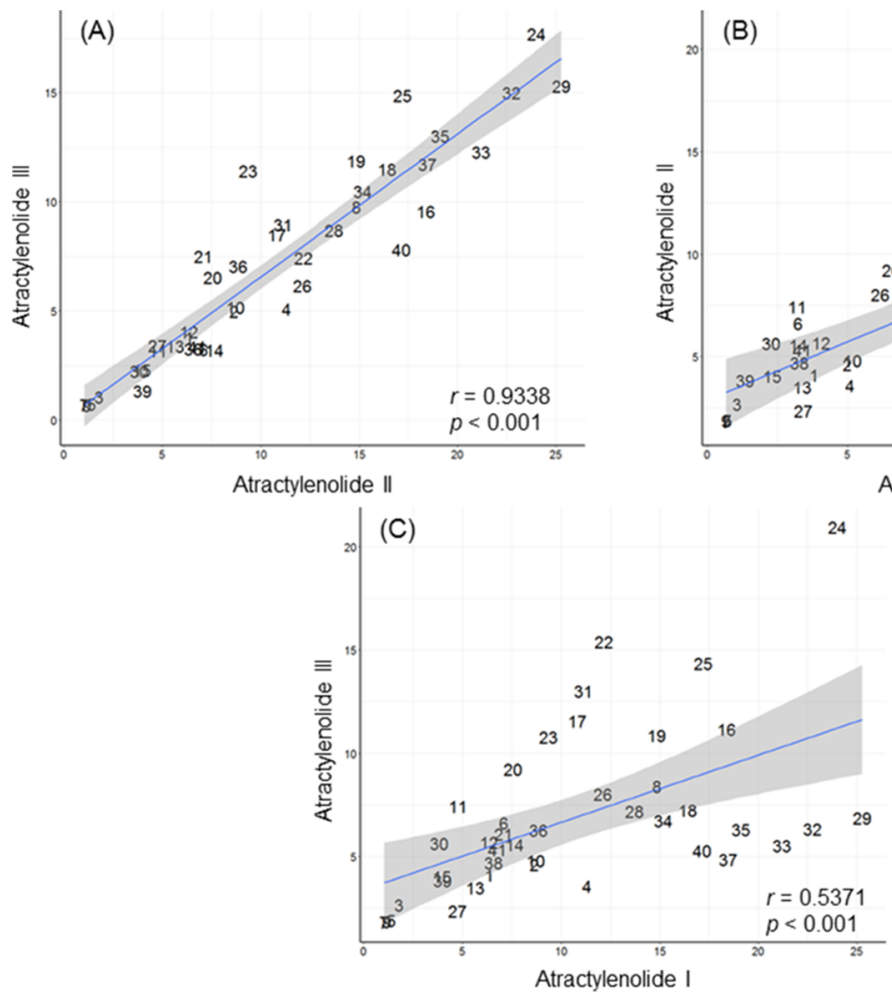

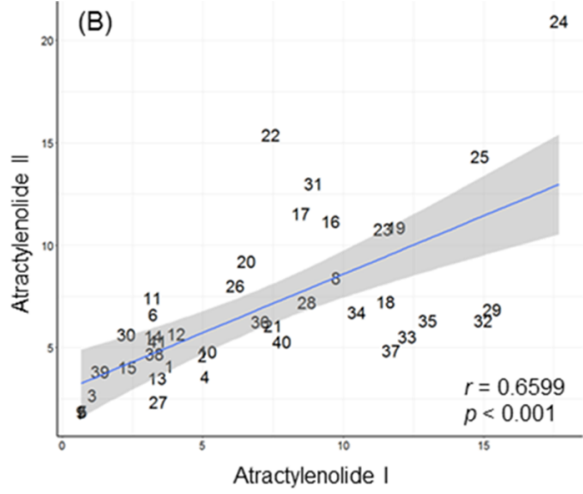
tylenolide 1

Figure 1. Pearson's correlation coefficients $(r)$ between the average contents of atractylenolide III, atractylenolide II, and atractylenolide I in the A. japonica samples. (A) The correlation between the content of atractylenolide III and atractylenolide II; (B) the correlation between the content of atractylenolide II and atractylenolide I; (C) the correlation between the content of atractylenolide III and atractylenolide I. All correlations were significant at $p$-values $<0.001$. Each $A$. japonica sample is represented as a sample number.

fragment ion $(m / z$ 201.98), which was consistent with previous results (Figure S2, Supporting Information). ${ }^{12,13,22-25}$ The fragmented product ions of each compound, $m / z$ 231.03, 215.07, and 185.09 for atractylenolides III, II, and I, respectively, were selected for identification and quantification of the marker compounds in the methanol extracts of $A$. japonica samples.

2.3. Method Validation. The correlation coefficients $\left(r^{2}\right)$ of each linear equation were in the range of $0.9976-0.9991$ within the linear ranges: $0.47-30.00 \mu \mathrm{g} / \mathrm{mL}$ for atractylenolide III, $0.31-20.00 \mu \mathrm{g} / \mathrm{mL}$ for atractylenolide II, and $0.47-30.00$ $\mu \mathrm{g} / \mathrm{mL}$ for atractylenolide I. LODs and LOQs were 23-43 and 78-146 ng/mL, respectively (Table S1, Supporting Information). The precision estimates of the LC/MS method determined for three concentrations of marker compounds were $1.97-12.32 \%$ of the relative standard deviation (RSD) value within one day and $3.19-12.03 \%$ of the RSD value during three days (Table 1). Recovery of added compounds ranged from 83.50 to $101.93 \%$, with the RSD value less than $14.00 \%$ (Table 2). The repeatability, represented as RSD values, was $4.63-6.04 \%$ for the absolute peak area and $0.06-$ $0.62 \%$ for the retention time (Table S2, Supporting Information).

These results indicate that the LC/MS method developed here allows linear, sensitive detection of each compound and that precise, accurate, and reliable measurements can be obtained for the quantification of the three marker compounds in the methanol extracts of $A$. japonica rhizomes.

2.4. Quantification of the Three Marker Compounds in A. japonica Samples. The fragmentation of protonated ion molecules to the most intense fragment ions of $\mathrm{m} / z 249.00$ $\rightarrow 231.03$ for atractylenolide III, $m / z 233.00 \rightarrow 215.07$ for atractylenolide II, and $m / z 231.00 \rightarrow 185.09$ for atractylenolide I was employed for quantification in A. japonica samples. The average content of the three marker compounds varied across the A. japonica samples. The amount of atractylenolide III was highest, followed by atractylenolides I and II in most samples with significance, which is consistent with the previous study, ${ }^{22}$ although the no. 25 sample showed no significant difference in contents. In addition, the differences in the amounts (highest amount/lowest amount) of each compound were from 11- to 26-fold within A. japonica samples (Table S3, Supporting Information).

A diverse range of environmental circumstances, including location, climate, wild/cultivated, seasons, and soil composition, is speculated to determine the production or accumulation of sesquiterpenes in Atractylodes rhizomes because the occurrence of secondary metabolites is mainly affected by such extrinsic factors. ${ }^{26-30}$ Moreover, the defense system of the Atractylodes rhizome against pathogens can also influence (mainly increase) sesquiterpene accumulation through the induction of jasmonic acid, which is a plant-signaling molecule in defense response. ${ }^{31}$ It is assumed that those environmental and defensive factors result in various contents of atractylenolides in A. japonica rhizomes in different locations.

2.5. Correlation between the Average Content of the Three Marker Compounds in A. japonica Samples. Correlations between the marker compounds based on the average content measured in $A$. japonica samples were evaluated by using Pearson's correlation coefficient $(r)$, which is a value that ranges from -1 to +1 . Pearson's correlation coefficient is generally used to evaluate the degree of linear interrelation between two variables and measures an influence of one variable on the other variable. ${ }^{32}$ 
Scheme 1. Tentative Pathway of the Biosynthesis of Atractylenolide I-III from Atractylon

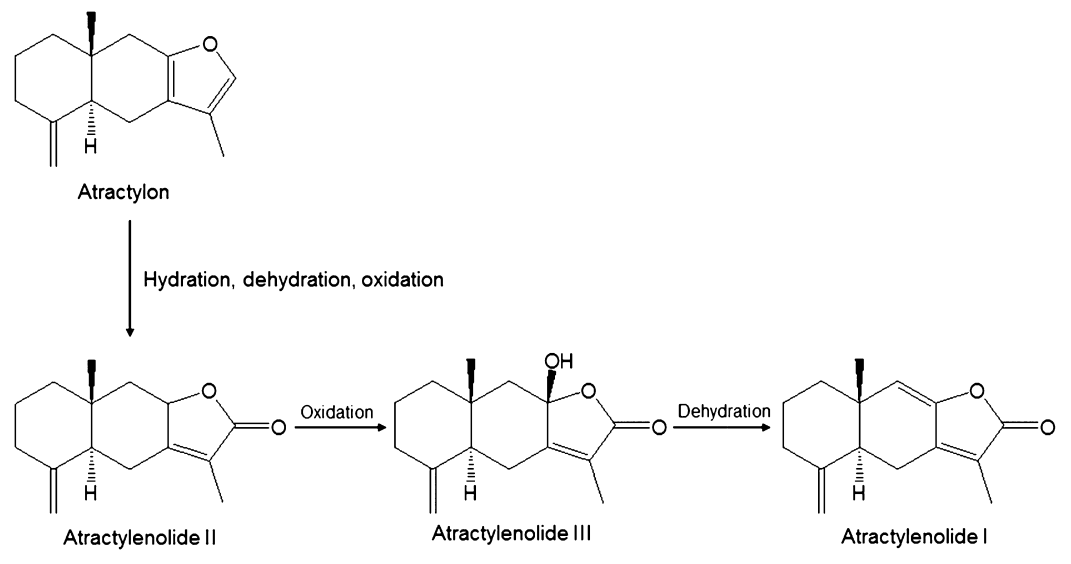

Scheme 2. Proposed Reaction Mechanism Adapted from the Catalytic Cycle of Cytochrome P450 for C-H Bond Activation Reaction

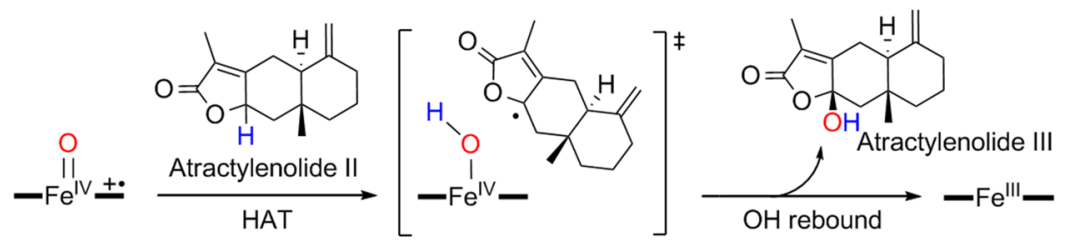

All pairs of average content of the three marker compounds showed positive correlation coefficients with significance $(p<$ $0.001)$; the pair of atractylenolide II-atractylenolide III exhibited a higher coefficient $(r>0.9)$ and the atractylenolide I-atractylenolide III pair had the lowest coefficient $(r>0.5)$, following that of atractylenolide I-atractylenolide II $(r>0.6)$ (Figure 1). Given that a higher coefficient value (approaching to +1 ) indicates stronger correlation and more linearity between two variables in a positive coefficient, ${ }^{33}$ the coefficients between the marker compounds indicate that an increase in the amount of one compound can be related to an increase in the amount of other compounds in A. japonica rhizomes. As the production of atractylenolides is the result of their biosynthesis in A. japonica rhizomes, higher correlation between the content of atractylenolides means that the existence of the compound is strongly related to the course of the biosynthetic pathway. This connectivity in the contents of compounds can be explained by their biosynthetic features. Atractylenolides I-III, originally derived from atractylon, are serially converted: during autoxidation, the C-8 hydrogen of atractylenolide II is oxidized to a C-8 hydroxyl group, which is then converted into atractylenolide III, which is dehydrated to atractylenolide I with a loss of hydroxyl group at C-8 as a water molecule (Scheme 1). ${ }^{34,35}$

2.6. Oxidative Transformation of Atractylenolide II Using the Biomimetic Model of Cytochrome P450. High-valent iron(IV)-oxo porphyrin $\pi$-cation radical species have frequently been invoked as key intermediates in the $\mathrm{O}_{2}$ activating catalytic cycles of heme iron enzymes, such as CYP450, catalases, and peroxidases. ${ }^{21,36,37}$ As CYP450 participates in the formation of diverse sesquiterpenoids in A. lancea, it is assumed that CYP450 enzymes can be found in plant $A$. japonica Koidzumi, ${ }^{38}$ thereby, allowing an alternative pathway of the oxidative transformation of atractylenolides because of the plausible formation of highly reactive Cpd I via $\mathrm{O}_{2}$-activation. On the basis of this important hypothesis, one might expect a hydrogen atom abstraction (HAT) from $\mathrm{C}-\mathrm{H}$ bonds of atractylenolides by Cpd I, followed by the hydroxyl rebound process that results in the hydroxyl group incorporation into atractylenolides (Scheme 2). ${ }^{21}$

To investigate this hypothesis, we first generated the iron(IV)-oxo porphyrin $\pi$-cation radical complex, $\left[\left(\mathrm{tmp}^{+\bullet}\right)\right.$ $\left.\mathrm{Fe}^{\mathrm{IV}}(\mathrm{O})\right]^{+}$(1) (tmp = meso-tetramesitylporphyrin) according to the literature procedures. Addition of 2.4 equiv of metachloroperbenzoic acid ( $m$-CPBA) to the mixture solvent of $\mathrm{CH}_{3} \mathrm{CN}$ and $\mathrm{CH}_{2} \mathrm{Cl}_{2}$ (9:1) containing $\left[\mathrm{Fe}{ }^{\mathrm{III}}(\mathrm{tmp})\right]\left(\mathrm{CF}_{3} \mathrm{SO}_{3}\right)$ resulted in the formation of 1 at $-40{ }^{\circ} \mathrm{C}$ (Figure 2a). We then explored the reactivities of $\mathbf{1}$ in the $\mathrm{C}-\mathrm{H}$ bond activation reaction of atractylenolides. Upon addition of atractylenolide II to the solution of 1 , the absorption band at $665 \mathrm{~nm}$ due to 1 disappeared with a concomitant increase of the absorption band at $505 \mathrm{~nm}$ because of $\left[\mathrm{Fe}^{\mathrm{III}}(\mathrm{tmp})\right]\left(\mathrm{CF}_{3} \mathrm{SO}_{3}\right)$ at $-40{ }^{\circ} \mathrm{C}$. The decay rate was increased linearly with the increase of atractylenolide II concentrations; second-order rate constants $\left(k_{2}\right)$ of $1.5 \times 10^{-1} \mathrm{M}^{-1} \mathrm{~s}^{-1}$ was determined (Figure $2 \mathrm{~b}$ ).

Very interestingly, the product analysis of the reaction solution of the oxidation of atractylenolide II by 1 revealed that atractylenolide III was produced as a sole product with a quantitative yield ( $>95 \%$ based on the amount of 1 used). When the atractylenolide II oxidation was performed with 1 in the presence of $\mathrm{H}_{2}{ }^{18} \mathrm{O}$, atractylenolide III contained $90 \%$ of ${ }^{18} \mathrm{O}$ under the identical reaction conditions (Figure 3). This result clearly indicates that the source of oxygen in the hydroxylated product was the iron(IV)-oxo porphyrin $\pi-$ cation radical species through oxygen exchange with ${ }^{18} \mathrm{O}$ labeled water. The conversion of atractylenolide III to atractylenolide I via dehydration was also detected when the resulting solution was kept under an air atmosphere for several hours.

We also characterized the iron product formed in the atractylenolide II oxidation by $\mathbf{1}$. The negative mode ESI-MS spectrum of the reaction solution exhibited a prominent peak 

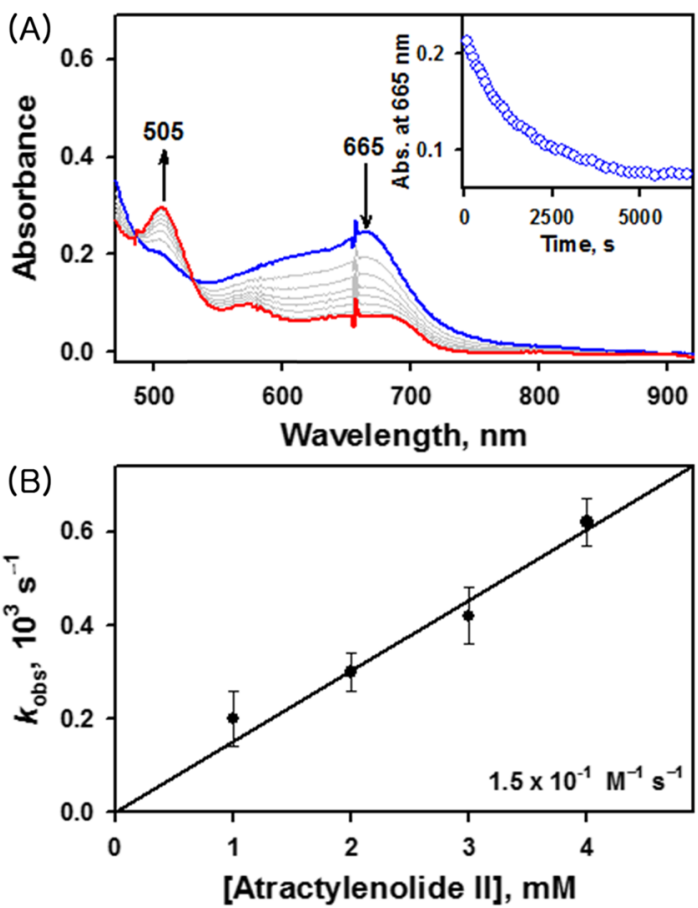

Figure 2. (A) UV-vis spectral changes of $\left[\left(\mathrm{tmp}^{+\bullet}\right) \mathrm{Fe}^{\mathrm{IV}}(\mathrm{O})\right]^{+}(0.025$ $\mathrm{mM}$ ) (1, blue line) upon addition of 40 equiv of atractylenolide II at $-40{ }^{\circ} \mathrm{C}$ resulting in the formation of $\left[\mathrm{Fe}^{\mathrm{III}}(\mathrm{tmp})\right]\left(\mathrm{CF}_{3} \mathrm{SO}_{3}\right)$ (red line). Inset shows the time course of the decay of 1 monitored at 665 nm. (B) Plot of pseudo-first order rate constants $\left(k_{\mathrm{obs}}\right)$ against the concentrations of atractylenolides II to determine $k_{2}$ in the oxidation of atractylenolide II by $\mathbf{1}$.

auth entic atractylen olide III
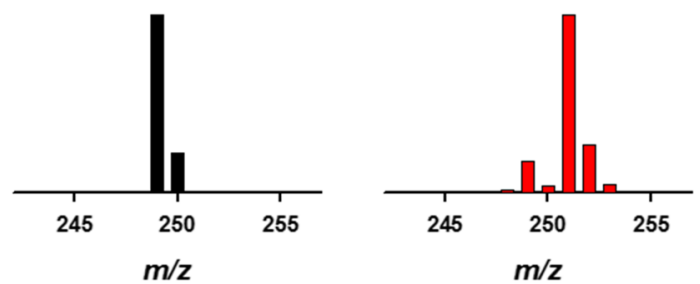

Figure 3. Mass distribution patterns of authentic atractylenolide III (left panel) and atractylenolide III (right panel) obtained in the reaction of 1 and atractylenolide $\mathrm{II}$ in the presence of $\mathrm{H}_{2}{ }^{18} \mathrm{O}$ in $\mathrm{CH}_{3} \mathrm{CN} / \mathrm{CH}_{2} \mathrm{Cl}_{2}(9: 1)$ at $-40{ }^{\circ} \mathrm{C}$ under an $\mathrm{Ar}$ atmosphere. The ${ }^{18} \mathrm{O}$ labeled products were $\sim 80 \%$.

at $m / z$ of 836.8 , whose mass and isotopic distribution patterns correspond to $[\mathrm{Fe}(\mathrm{tmp})]^{+}$(calculated $\mathrm{m} / z$ of 836.9 ) (Figure 4). Taken together, the hydroxylation of atractylenolide II by 1 proceeded via HAT from the $\mathrm{C}-\mathrm{H}$ bond of atractylenolide II, followed by an oxygen rebound process, resulting in the formation of $\left[\mathrm{Fe}^{\mathrm{III}}(\mathrm{tmp})\right]^{+}$and atractylenolide III. Dehydration of atractylenolide III to atractylenolide I was also observed when the resulting reaction solution was kept for several hours under an air atmosphere.

\section{CONCLUSIONS}

An efficient, accurate, and precise analytical LC/ion-trap MS method was developed and applied for the quantification of atractylenolide I, II, and III in A. japonica rhizomes with a validation in terms of linearity, recovery, precision, and repeatability. The positive ionization mode was employed for

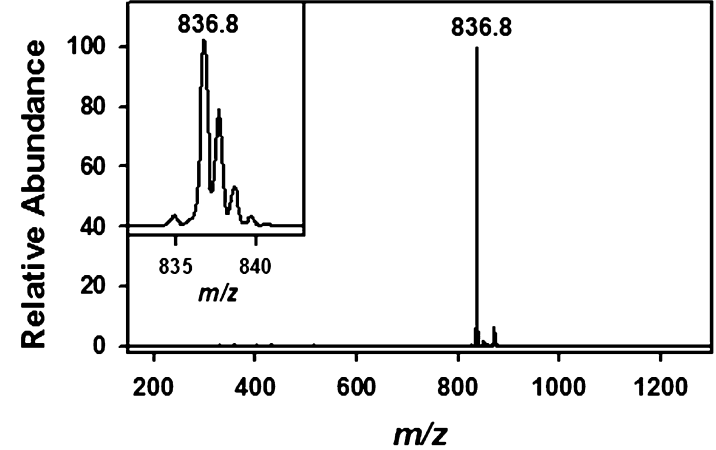

Figure 4. ESI MS spectra of the reaction solution taken after the complete reaction between $1(0.050 \mathrm{mM})$ and atractylenolide II (40 equiv) in positive mode recorded in $\mathrm{CH}_{3} \mathrm{CN}$ at $-40{ }^{\circ} \mathrm{C}$. The peaks at $m / z$ of 836.8 are assigned as $[\mathrm{Fe}(\mathrm{tmp})]^{+}$(calculated $m / z$ of 836.9 ). Inset shows the isotopic distribution patterns of $[\mathrm{Fe}(\mathrm{tmp})]^{+}$.

the detection of molecular ion fragmentation at $\mathrm{m} / z 249.00 \rightarrow$ 231.03 for atractylenolide III, $\mathrm{m} / z 233.00 \rightarrow 215.07$ for atractylenolide II, and $m / z 231.00 \rightarrow 185.09$ for atractylenolide $\mathrm{I}$. The average contents of the three atractylenolides were determined in A. japonica samples obtained from different locations in Korea and China. The results reveal a variation in the amounts of analytes among the samples, and high correlations were observed between the content of the atractylenolides in the samples. A significant correlation between atractylenolides was evidenced by the CYP450mimetic oxidation model. Finally, we also provided (i) kinetic value of oxidative transformation of atractylenolide II by highvalent iron(IV)-oxo porphyrin $\pi$-cation radical species and (ii) experimental evidence that the hydroxylation of atractylenolide II occurs via hydrogen atom abstraction, followed by an oxygen rebound mechanism. These results would be helpful to understand the existence and role of oxidative enzymes, especially cytochrome P450, during the biosynthesis in the A. japonica plant.

\section{EXPERIMENTAL SECTION}

4.1. Chemicals and Reagents. Commercially available reagents were of the best available purity and were used without further purification unless otherwise noted. LC/MSgrade acetonitrile, water, and formic acid were purchased from Fisher Scientific (Pittsburgh, PA, USA). HPLC-grade methanol was obtained from J.T. Baker Inc. (Phillipsburg, NJ, USA). Acetonitrile $\left(\mathrm{CH}_{3} \mathrm{CN}\right)$ and dichloromethane $\left(\mathrm{CH}_{2} \mathrm{Cl}_{2}\right)$ were dried according to the literature procedures and distilled under Ar prior to use. ${ }^{39} \mathrm{~m}$-CPBA was purified by washing with phosphate buffer ( $\mathrm{pH}$ 7.4) followed by water and then dried under reduced pressure. ${ }^{39}\left[\mathrm{Fe}^{\mathrm{III}}(\mathrm{tmp})\right] \mathrm{Cl}$ was purchased from Frontier Scientific Inc. (Logan, UT, USA). [Fe $\left.{ }^{\mathrm{III}}(\mathrm{tmp})\right]$ $\left(\mathrm{CF}_{3} \mathrm{SO}_{3}\right)$ was prepared by stirring equimolar amounts of $\left[\mathrm{Fe}^{\mathrm{III}}(\mathrm{tmp})\right] \mathrm{Cl}$ and $\mathrm{AgCF}_{3} \mathrm{SO}_{3}$, followed by filtration through a $0.45 \mathrm{~mm}$ filter; the resulting solution was used immediately. ${ }^{40}$ Atractylenolides I and III were purchased from Biomart (Guangzhou, Guangdong, China). Atractylenolide II was purchased from Chengdu Biopurify Phytochemicals (Chengdu, Sichuan, China). Bergapten (IS) was purchased from ChemFace (Wuhan, Hubei, China). All marker compounds had purities of $\geq 98 \%$.

Forty-one samples of $A$. japonica rhizomes were either purchased from herbal companies or harvested from the wild 
fields in Korea and China. The samples were authenticated by the authors (Table S4, Supporting Information). Voucher specimens (2016-PNUKMAJ-01-41) have been deposited at the School of Korean Medicine, Pusan National University.

4.2. Sample Preparation. The A. japonica rhizomes were pulverized and passed through a $500 \mu \mathrm{m}$ testing sieve (Chunggyesanggong-sa; Gunpo, Gyeonggi, Korea) to produce a homogenized powder. Accurately weighed powder $(0.5 \mathrm{~g})$ was extracted into $10 \mathrm{~mL}$ of methanol $(\mathrm{w} / \mathrm{v})$ for $30 \mathrm{~min}$ using an ultrasonic extractor (Power Sonic 520; Hwashin Tech, Daegu, Korea). The extracted solution was centrifuged at 10 $000 \mathrm{rpm}$ for $5 \mathrm{~min}$. The supernatant was transferred to a 1.5 $\mathrm{mL}$ polypropylene tube and then evaporated using a nitrogenblowing concentrator (MGS2200; EYELA, Miyagi, Japan). The concentrated extract was dissolved in HPLC-grade methanol to a concentration of $1000 \mu \mathrm{g} / \mathrm{mL}$ and filtered through a $0.2 \mu \mathrm{m}$ syringe filter (BioFact, Daejeon, Korea) prior to LC/MS injection.

4.3. Analytical Apparatus. An Accela LC system (Thermo Fisher Scientific; CA, USA) equipped with a refrigerated autosampler, degasser, and quaternary solvent pump was employed for the quantitative analysis. The three marker compounds were separated on a Hypersil GOLD $\mathrm{C}_{18}$ column $(2.1 \mathrm{~mm} \times 100 \mathrm{~mm}, 1.9 \mu \mathrm{m}$; Thermo Fisher Scientific, CA, USA) at $35{ }^{\circ} \mathrm{C}$. The flow rate was $250 \mu \mathrm{L} / \mathrm{min}$, and the injection volume was $5 \mu \mathrm{L}$. The mobile phase consisted of water (containing $0.1 \%$ formic acid; A) and acetonitrile (B), and an isocratic elution was performed with an $\mathrm{A} / \mathrm{B}$ ratio of 45:55 (v/v) until the end of the analysis (to $15 \mathrm{~min}$ ).

An LCQ Fleet ion-trap mass spectrometer (Thermo Fisher Scientific; CA, USA) was used to analyze the compounds in the LC eluent, which was introduced into the ESI source in positive-ion mode. Instrumental conditions were as follows: sheath gas (nitrogen), 50 arb units; auxiliary gas (nitrogen), 20 arb units; spray voltage, $5.0 \mathrm{kV}$, capillary temperature, $280{ }^{\circ} \mathrm{C}$; and capillary voltage, $30.0 \mathrm{~V}$. Tandem mass scans $\left(\mathrm{MS}^{2}\right)$ from the protonated molecular ion $[\mathrm{M}+\mathrm{H}]^{+}$were employed for quantification of the marker compounds and the IS. The collision energy was set at $35 \%$ for collision-induced dissociation in the $\mathrm{MS}^{2}$ experiment. Data were processed using Xcalibur software (v. 2.1.0; Thermo Fisher Scientific, CA, USA).

UV-vis spectra were recorded on a Hewlett Packard Agilent 8454 UV-visible spectrophotometer equipped with a Unisoku cryostat system (USP-203; Unisoku, Japan). Product analysis was performed with Agilent Technologies 1220 Infinity II HPLC.

4.4. Method Validation. The three marker compounds were accurately weighed and dissolved in methanol at 1000 $\mu \mathrm{g} / \mathrm{mL}$ to make stock solutions, which were diluted to produce seven working solutions containing the IS. The calibration curves were constructed by plotting the peak area ratio of the three marker compounds to the IS ( $y$-axis) versus the concentration of the three marker compounds in the working solution ( $x$-axis). The linearity of each calibration curve was evaluated by the correlation coefficients $\left(r^{2}\right)$. LOD and LOQ were determined as signal-to-noise $(\mathrm{S} / \mathrm{N})$ ratios of 3 and 10 , respectively. Precision was determined by analyzing low, middle, and high concentrations of working solutions of the marker compounds three times within a day (intraday precision) and during three consecutive days (interday precision). Values of precision are indicated as RSD, as follows: $\operatorname{RSD}(\%)=[($ standard deviation $/$ mean $) \times 100]$. The accuracy of the LC/MS method used was evaluated by recovery testing. Three known amounts of marker compounds (low, middle, and high) were added to the methanol extract of A. japonica samples, and the recovery was calculated as follows: recovery $(\%)=[$ (detected concentration - initial concentration)/spiked concentration] $\times 100$.

4.4.1. Repeatability. The repeatability was indicated as RSD values of retention times and RSD values of the absolute areas of the marker compounds, based on six replicate analyses of a sample.

4.5. Statistical Analysis. Multiple comparisons between the contents of atractylenolide I-III in each A. japonica sample were performed by using Tukey's test. Differences were considered significant at $p<0.05, p<0.01$, or $p<0.001$. Pearson's correlation coefficient of the average content of atractylenolide I-III in the A. japonica samples was calculated with significance of $p$-values $<0.001$. Multiple comparisons and Pearson's correlation coefficient were calculated by using open-source software R (v. 3.4.3; The R Foundation for Statistical Computing).

4.6. Kinetics Studies. Reactions were followed by monitoring UV-vis spectral changes of reaction solutions at $-40{ }^{\circ} \mathrm{C}$. All reactions were run, at least, in triplicates, and the data reported represent the average of these reactions. Iron(IV)-oxo porphyrin $\pi$-cation radical species, [( $\left.\mathrm{tmp}^{+\bullet}\right)$ $\left.\mathrm{Fe}^{\mathrm{IV}}(\mathrm{O})\right]^{+}(\mathbf{1})$, was prepared by literature methods. ${ }^{40}$ The formation of the iron-oxo intermediates was confirmed by UV-vis spectroscopy. Subsequently, appropriate amounts of atractylenolie II were added to the reaction solutions. After the completion of reactions, pseudo-first-order fitting of the kinetic data allowed us to determine $k_{\text {obs }}$ values. The resulting solution was directly injected to HPLC in order to identify and quantify the reaction products. Products were determined by comparing retention times and mass patterns to those of known authentic samples. Atractylenolide III (>95\%) was formed as a sole product. Product yields were determined by comparison against standard curves prepared with authentic samples.

\section{ASSOCIATED CONTENT}

\section{S Supporting Information}

The Supporting Information is available free of charge on the ACS Publications website at DOI: 10.1021/acsomega.8b02005.

Tables S1-S4 showing calibration curves, repeatability, and the average contents of atractylenolide I, II, and III and the sample list of $A$. japonica rhizomes and figures S1 and S2 showing extracted ion chromatograms of atractylenolide I, II, and III and their mass spectra (PDF)

\section{AUTHOR INFORMATION}

\section{Corresponding Authors}

*E-mail: kmsct@pusan.ac.kr. Phone: +82 51510 8456. Fax: +82510510 8420 (J.-H.K.).

*E-mail: hsw@sm.ac.kr. Phone: +82 22077 7829. Fax: +82 2 20777829 (S.H.).

ORCID $\odot$

Jung-Hoon Kim: 0000-0002-9704-0206

Seungwoo Hong: 0000-0001-7953-8433

Notes

The authors declare no competing financial interest. 


\section{ACKNOWLEDGMENTS}

This work was supported by the National Research Foundation of Korea (NRF) grant funded by the Korea government through MSIP (NRF-2015R1C1A1A01053466 to J.-H.K.), (NRF-2017R1C1B2002037 to S.H.), and Sookmyung Women's University Grants (1-1703-2038 to S.H.).

\section{REFERENCES}

(1) Choi, K. H.; Jeong, S. I.; Lee, J. H.; Hwang, B. S.; Kim, S. J.; Lee, S.; Choi, B. K.; Jung, K. Y. Pharmacological mechanism responsible for the Atractylodes japonica-induced distal colonic contraction in rats. Phytomedicine 2011, 18, 408-413.

(2) Park, J. J.; Chon, N. R.; Lee, Y. J.; Park, H. The effects of an extract of Atractylodes japonica rhizome, SKI3246 on gastrointestinal motility in guinea pigs. J. Neurogastroenterol. Motil. 2015, 21, 352360.

(3) Liu, Y.; Jia, Z.; Dong, L.; Wang, R.; Qiu, G. A randomized pilot study of atractylenolide I on gastric cancer cachexia patients. EvidenceBased Complementary Altern. Med. 2008, 5, 337-344.

(4) Wang, K.-T.; Chen, L.-G.; Wu, C.-H.; Chang, C.-C.; Wang, C.C. Gastroprotective activity of atractylenolide III from Atractylodes ovata on ethanol-induced gastric ulcer in vitro and in vivo. J. Pharm. Pharmacol. 2010, 62, 381-388.

(5) Ma, L.; Mao, R.; Shen, K.; Zheng, Y.; Li, Y.; Liu, J.; Ni, L. Atractylenolide I-mediated Notch pathway inhibition attenuates gastric cancer stem cell traits. Biochem. Biophys. Res. Commun. 2014, $450,353-359$.

(6) Song, H.-P.; Hou, X.-Q.; Li, R.-Y.; Yu, R.; Li, X.; Zhou, S.-N.; Huang, H.-Y.; Cai, X.; Zhou, C. Atractylenolide I stimulates intestinal epithelial repair through polyamine-mediated $\mathrm{Ca}^{2+}$ signaling pathway. Phytomedicine 2017, 28, 27-35.

(7) Chen, Q.; Li, P.; Zheng, F.; He, J.; Yi, Y. Validated method for the quantification of atractylenolide III in different processed products of Rhizoma Atractylodes Macrocephalae. Phytochem. Anal. 2011, 22, $10-13$.

(8) Sun, X. H.; Ge, J. Simultaneous determination of atractylenolide II and III in rhizoma Atractylodes Macrocephalae and Chinese medicinal preparation by reverse-phase high-performance liquid chromatography. In Frontier and Future Development of Information Technology in Medicine and Education; Li, S., Jin, Q., Jiang, X., Park, J., Eds.; Springer: Dordrecht, 2014; pp 1283-1289.

(9) Yun, B. R.; Weon, J. B.; Lee, B.; Lee, J.; Eom, M. R.; Ma, C. J. Quantitative analysis of atractylenolides I and III in Atractylodes japonica. Korean J. Pharmacogn. 2013, 44, 53-59.

(10) Chen, Q.; Li, P.; Yang, H.; Zhu, J. Sensitive capillary GC-MSSIM determination of atractylenolide I and atractylenolide III in Atractylodes macrocephala. Anal. Lett. 2009, 42, 2547-2555.

(11) Liao, Q. G.; Li, R. L.; Luo, L. G. Accelerated solvent extraction coupled to GC-MS/MS for the analysis of atractylenolide in Atractylodes macrocephala. Chromatographia 2012, 75, 931-935.

(12) Cho, H.-D.; Kim, U.; Suh, J. H.; Eom, H. Y.; Kim, J.; Lee, S. G.; Choi, Y. S.; Han, S. B. Classification of the medicinal plants of the genus Atractylodes using high-performance liquid chromatography with diode array and tandem mass spectrometry detection combined with multivariate statistical analysis. J. Sep. Sci. 2016, 39, 1286-1294.

(13) Chen, Q.; He, H.; Li, P.; Zhu, J.; Xiong, M. Identification and quantification of atractylenolide I and atractylenolide III in Rhizoma Atractylodes Macrocephala by liquid chromatography-ion trap mass spectrometry. Biomed. Chromatogr. 2013, 27, 699-707.

(14) Bansal, S. S.; Abbate, V.; Bomford, A.; Halket, J. M.; Macdougall, I. C.; Thein, S. L.; Hider, R. C. Quantitation of hepcidin in serum using ultra-high-pressure liquid chromatography and a linear ion trap mass spectrometer. Rapid Commun. Mass Spectrom. 2010, 24, $1251-1259$.

(15) Dolman, S.; Pelzing, M. An optimized method for the determination of perfluorooctanoic acid, perfluorooctane sulfonate and other perfluorochemicals in different matrices using liquid chromatography/ion-trap mass spectrometry. J. Chromatogr. B 2011, $879,2043-2050$

(16) Chen, X.-F.; Wu, H.-T.; Tan, G.-G.; Zhu, Z.-Y.; Chai, Y.-F. Liquid chromatography coupled with time-of-flight and ion trap mass spectrometry for qualitative analysis of herbal medicines. J. Pharm. Anal. 2011, 1, 235-245.

(17) Jing, J.; Chan, C.-o.; Xu, L.; Jin, D.; Cao, X.; Mok, D. K. W.; Parekh, H. S.; Chen, S. Development of an in-line HPLC fingerprint ion-trap mass spectrometric method for identification and quality control of Radix Scrophulariae. J. Pharm. Biomed. Anal. 2011, 56, $830-835$.

(18) Chen, Q.; Zhang, Y.; Zhang, W.; Chen, Z. Identification and quantification of oleanolic acid and ursolic acid in Chinese herbs by liquid chromatography-ion trap mass spectrometry. Biomed. Chromatogr. 2011, 25, 1381-1388.

(19) Chen, Q.; Zhang, W.; Zhang, Y.; Chen, J.; Chen, Z. Identification and quantification of active alkaloids in Catharanthus roseus by liquid chromatography-ion trap mass spectrometry. Food Chem. 2013, 139, 845-852.

(20) Weitzel, C.; Simonsen, H. T. Cytochrome P450-enzymes involved in the biosynthesis of mono- and sesquiterpenes. Phytochem. Rev. 2015, 14, 7-24.

(21) de Montellano, P. R. O. Hydrocarbon hydroxylation by cytochrome P450 enzymes. Chem. Rev. 2011, 110, 932-948.

(22) Yan, H.; Sun, Y.; Zhang, Q.; Yang, M.; Wang, X.; Wang, Y.; Yu, Z.; Zhao, Y. Simultaneous determination and pharmacokinetic study of Atractylenolide I, II and III in rat plasma after intragastric administration of Baizhufuling extract and Atractylodis extract by UPLC-MS/MS. J. Chromatogr. B 2015, 993-994, 86-92.

(23) Shi, Y.-y.; Guan, S.-h.; Tang, R.-n.; Tao, S.-j.; Guo, D.-a. Simultaneous determination of atractylenolide II and atractylenolide III by liquid chromatography-tandem mass spectrometry in rat plasma and its application in a pharmacokinetic study after oral administration of Atractylodes Macrocephala Rhizoma extract. Biomed. Chromatogr. 2012, 26, 1386-1392.

(24) Shan, G.-S.; Zhang, L.-X.; Zhao, Q.-M.; Xiao, H.-B.; Zhuo, R. J.; Xu, G.; Jiang, H.; You, X.-M.; Jia, T.-Z. Metabolomic study of raw and processed Atractylodes macrocephala Koidz by LC-MS. J. Pharm. Biomed. Anal. 2014, 98, 74-84.

(25) Huo, H. L.; Yu, S. H.; Liu, X. Z.; Meng, Y.; Ren, Y. P.; Zhang, L. T. Simultaneous and sensitive determination of eight coumarins in rat bile and urine after oral administration of Radix Angelicae Dahuricae extract by liquid chromatography-electrospray ionization-mass spectrometry. Acta Chromatogr. 2013, 25, 201-219.

(26) Takeda, O.; Miki, E.; Terabayashi, S.; Okada, M.; Lu, Y.; He, H.-S.; He, S.-A. A comparative study on essential oil components of wild and cultivated Atractylodes lancea and A. chinensis. Planta Med. 1996, 62, 444-449.

(27) Takeda, O.; Miki, E.; Terabayashi, S.; Okada, M.; He, S.-a.; Sashida, Y. Seasonal variation of essential oil components in Atractylodes lancea (Thunb.) DC. propagated by division of their rhizomes. Chem. Pharm. Bull. 1996, 44, 823-828.

(28) Nakai, Y.; Yano, K.; Shiba, M.; Kondo, K.; Takeda, O.; Sakakibara, I.; Terabayashi, S.; Takeda, S.; Okada, M. Chemical characterization of rhizomes of Atractylodes lancea and A. chinensis identified by ITS sequences of nrDNA. J. Jpn. Bot. 2006, 81, 63-74.

(29) Yuan, Y.; Liu, Y. J.; Huang, L. Q.; Cui, G. H.; Fu, G. F. Soil acidity elevates some phytohormone and $\beta$-eudesmol contents in roots of Atractylodes lancea. Russ. J. Plant Physiol. 2009, 56, 133-137.

(30) Ouyang, Z.; Zhang, L.; Zhao, M.; Wang, P.; Wei, Y.; Fang, J. Identification and quantification of sesquiterpenes and polyacetylenes in Atractylodes lancea from various geographical origins using GC-MS analysis. Rev. Bras. Farmacogn. 2012, 22, 957-963.

(31) Ren, C.-G.; Dai, C.-C. Jasmonic acid is involved in the signaling pathway for fungal endophyte-induced volatile oil accumulation of Atractylodes lancea plantlets. BMC Plant Biol. 2012, 12, 128.

(32) Mudelsee, M. Estimating Pearson's correlation coefficient with bootstrap confidence interval from serially dependent time series. Math. Geol. 2003, 35, 651-665. 
(33) Mukaka, M. M. Statistics corner: A guide to appropriate use of correlation coefficient in medical research. Malawi Med. J. 2012, 24, 69-71.

(34) Hikino, H.; Hikino, Y.; Yosioka, I. Studies on the constituents of Atractylodes IX. Structure and autoxidation of atractylon. Chem. Pharm. Bull. 1964, 12, 755-760.

(35) Wang, X. F.; Wang, F.; Zhang, Y. H.; Zheng, X. H. Kinetics and mechanism studies on oxidizing reaction of atractylon in essential oil from Atractylodes macrocephala Koidz. Chin. J. Appl. Chem. 2007, 24, 301-305.

(36) Huang, Y.; Bie, Z.; Liu, Z.; Zhen, A.; Wang, W. Protective role of proline against salt stress is partially related to the improvement of water status and peroxidase enzyme activity in cucumber. Soil Sci. Plant Nutr. 2009, 55, 698-704.

(37) Kibinza, S.; Bazin, J.; Bailly, C.; Farrant, J. M.; Corbineau, F.; El-Maarouf-Bouteau, $\mathrm{H}$. Catalase is a key enzyme in seed recovery from ageing during priming. Plant Sci. 2011, 181, 309-315.

(38) Chen, F.; Wei, Y.-X.; Zhang, J.-M.; Sang, X.-M.; Dai, C.-C. Transcriptomics analysis investigates sesquiterpenoids accumulation pattern in different tissue of Atractylodes lancea (Thunb.) DC. plantlet. Plant Cell Tissue Organ Cult. 2017, 130, 73-90.

(39) Armarego, W. L. F.; Chai, C. L. L. Purification of Laboratory Chemicals, 6th ed.; Pergamon Press: Oxford, 2009.

(40) Kang, Y.; Chen, H.; Jeong, Y. J.; Lai, W.; Bae, E. H.; Shaik, S.; Nam, W. Enhanced Reactivities of Iron(IV)-Oxo Porphyrin $\pi$-Cation Radicals in Oxygenation Reactions by Electron-Donating Axial Ligands. Chem.-Eur. J. 2009, 15, 10039-10046. 